D I A L O G UES

IN MUSIC THERAPY

EDUCATION

\title{
Advanced methods of music therapy practice: Analytical music therapy, the Bonny Method of Guided Imagery and Music, Nordoff-Robbins music therapy, and vocal psychotherapy.
}

Cohen, N. S. (2018). Jessica Kingsley Publishers. 248 Pages.

Paperback, \$34.99. ISBN: 978-1-84905-776-9.

Kayla C. Daly MA, MT-BC, LMHC

Worcester Center for Expressive Therapies

Worcester, MA

KaylaDaly@WCETherapies.com

Keywords: music therapy education, advanced clinical techniques 
Nicki Cohen wrote Advanced Methods of Music Therapy Practice: Analytical Music Therapy, the Bonny Method of Guided Imagery and Music, Nordoff-Robbins Music Therapy, and Vocal Psychotherapy, to pay homage to her mentor Helen Bonny, and as a meaningful pursuit in educating music therapy clinicians to consider the pursuance of completing advanced training methods. Cohen gives a brief history of her own personal journey into the field of music therapy and how her initial training process left her wanting more. Her process from new music therapy clinician within the United States, to music therapy professor, to finally becoming a primary trainer within the Bonny Method of Guided Imagery and Music, lends itself to an unfolding search for understanding depth of practice and meaningful growth as a music therapist.

The goal of the book is to address seven questions posed by Cohen within the preface and revisited once again in Section III (or Chapter 8). These questions include how the method was developed, what have become its most remarkable features, how has it changed over time, and finally, what the future of the method looks like and how it relates to the field of music therapy (Cohen, 2018). The questions were posed to experts of each advanced music therapy method as a qualitative inquiry. Cohen produced case studies and completed a transcription of their responses that were then analyzed through the NVivo software program (2018). Cohen then wrote in-depth narratives for each of the founders' personal life experiences.

The narratives present the reader with a personal storyline that stimulates investment in the further reading of each method's techniques. Cohen's analysis of the four methods is valuable in the initial exploration of theory development, research, and clinical practice. The appraisal of techniques that pertain to each method are presented 
clearly and concisely. Similar to the processes of Mary Priestly and Helen Bonny, Cohen explains that it took her 30 years to truly discover "the unleashed power of music therapy" (Cohen 2018, p.13). Through her writing, Cohen educates new professionals so they may consider pursuing advanced methods training earlier on in their careers. The succinct manner in which the methods are presented may serve as a jumping off point for music therapy students in learning about European/American advanced methods of practice.

Chapters one and two are brief historical overviews of the early evolution of music therapy as a solidified professional practice and discipline within the United States as well as within the United Kingdom. Cohen's choice for highlighting these two countries was that two of the methods were developed within the United States, and the other two were developed within the United Kingdom. Chapter three presents definitions for the terms "theory," "model," and "method," and expands these definitions to encompass her rationale for choosing the terms to describe the four music therapy methods addressed within the text. It is important to note she addresses a connection and distinction between the definitions of advanced music therapy methods and advanced levels of practice. In the current pursuance of advanced music therapy methods, most music therapists are veteran clinicians who decide to deepen their training later on in their careers (Cohen, 2018).

Section II of the book includes Chapters 4-7, each one highlighting the work of a founder and their respective method. Each chapter consists of a uniform structure that reviews the founder's story and the various techniques, trainings, and writings associated with their method. This structure provides an extensive yet qualitatively 
unique approach to learning about the historical context of the method and how the method has impacted training and research. It concludes with a summary concerning how the method may proceed to grow in the future.

Cohen's work is different from other publications that address advanced methods of practice in that it presents a brief through line of the development, influence, and trends of the four most currently prolific models. The comprehensive comparisons of the methods provide a concise understanding of each method's techniques. Of these interviews, only Diane Austin, the original founder of Vocal Psychotherapy was available for direct contact interviewing. This newer method was included by Cohen in order to compare the older methods to that of a newer method (2018).

In the third and final Section, Cohen returns to her research questions to present her findings. The wonderfully refreshing aspect of this presentation is the way in which the responses from the experts vary. Major emergent themes include music as the core of each method, interpersonal and intrapersonal growth that occurs through the advanced training associated with the methods, the challenges that come with method development, and the establishment of relevant outcomes (Cohen 2018). Most of the experts report advanced training expanding their ability to move beyond the method itself, creating their own adaptations of its various techniques and interventions (Cohen 2018). Experts differ on how they perceive the founders' intentions in creating a new method, but what appears to resonate with them most is the various ways in which their relationship with the method has changed over time (Cohen 2018). Importantly, Cohen's findings reflect that while the methods are all very different in their techniques and philosophical or psychological foundations, there is value in each method and in their 
contributions to the field of music therapy. All experts reported feelings that the methods were now a part of music therapy practice (Cohen 2018). The author's gentle summary of the four methods provides a thoughtful overview of American/European clinical music therapy while also revealing a need for diversity, inclusion, and expansion among these advanced methods of practice.

Cohen's work presents a need for educators and faculty advisors to consider how they inspire students early on in their academic experiences. For Cohen, emphasis on clinical identity development and each student's own clinical philosophies with respect to music are influential in this process. The deepening of the student's intrapersonal relationship to music relates specifically to their understanding of advanced practice and its relevance to the future of the field. Curriculum development concerning the ontology of music in music therapy and how advanced methods transform clinical practice are of great importance in providing students with a diverse understanding of the work. Students may develop deepened interests in advanced studies and methods, ensuring that the future of music therapy clinical practice will continue to grow. American/European advanced methods have been of great value to the growth of the field and their overview, presented here, also reveals the need for greater diversity concerning what music therapists define as advanced practice methods. Cohen's book offers promise that additional research may challenge our understanding of method development and influence, calling attention to historically relevant methods beyond the scope of the text. 